Supporting Information for $\boldsymbol{A C S}$ Nano

\title{
Vertical Chemical Vapor Deposition Growth of Highly Uniform 2D Transition Metal Dichalcogenides
}

Lei Tang ${ }^{1}$, Tao $\mathrm{Li}^{2}$, Yuting Luo ${ }^{1}$, Simin Feng ${ }^{1}$, Zhengyang Cai ${ }^{1}$, Hang Zhang ${ }^{2}$, Bilu

$$
\text { Liu }^{1, *} \text {, Hui-Ming Cheng }{ }^{1,3^{*}}
$$

${ }^{1}$ Shenzhen Geim Graphene Center, Tsinghua-Berkeley Shenzhen Institute and Tsinghua Shenzhen International Graduate School, Tsinghua University, Shenzhen, Guangdong, 518055, P. R. China ${ }^{2}$ Institute of Engineering Thermophysics, Chinese Academy of Sciences, Beijing, 100190, P. R. China ${ }^{3}$ Shenyang National Laboratory for Materials Sciences, Institute of Metal Research, Chinese Academy of Sciences, Shenyang, Liaoning, 110016, P. R. China

Correspondence should be addressed to bilu.liu@sz.tsinghua.edu.cn,hmcheng@sz.tsinghua.edu.cn 
a

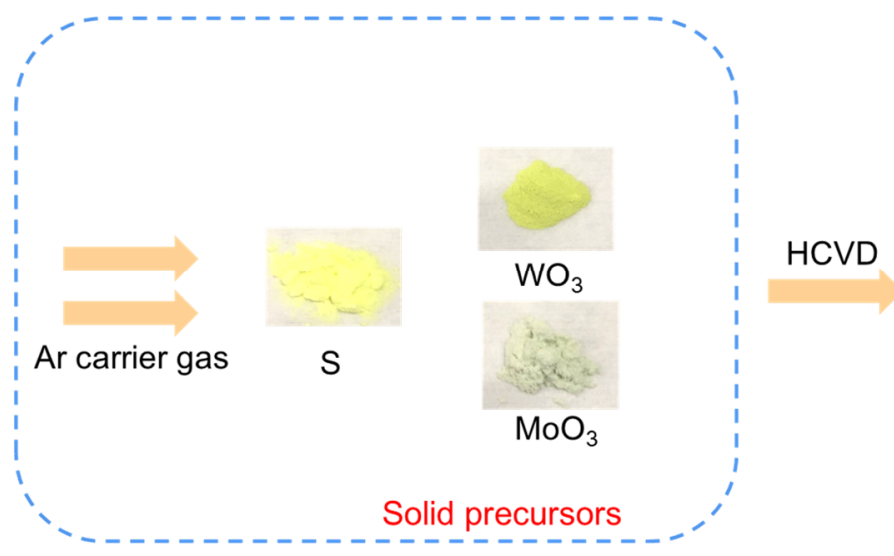

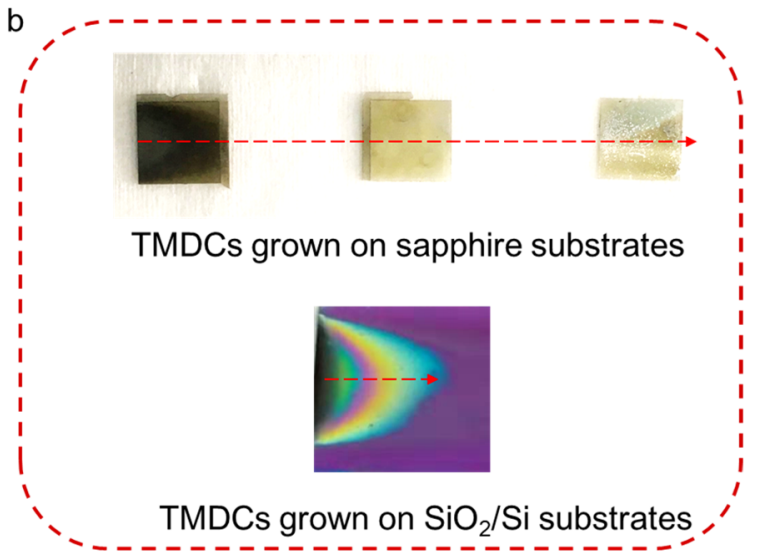

Figure S1. Photographs of (a) the solid precursors for TMDC growth, and (b) as-grown TMDCs show a gradient distribution in the growth profiles in a HCVD system with solid precursors, stemming from uncontrolled concentration, gas and temperature issues.

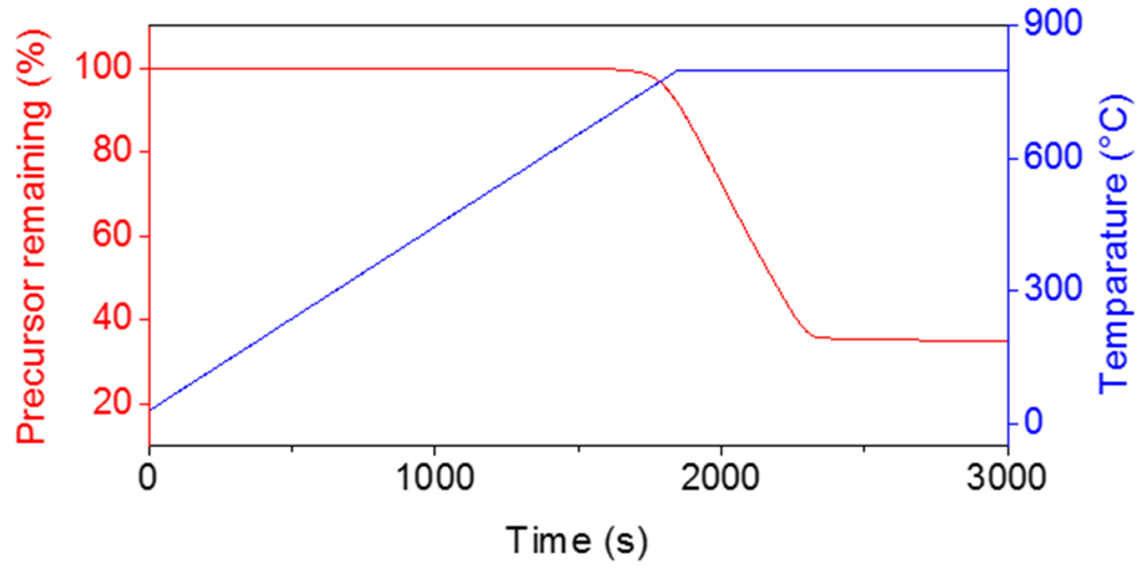

Figure S2. Thermogravimetric analysis (TGA) of the mass of metal oxide $\left(\mathrm{MoO}_{3}\right)$ reducing with increasing temperature and time. Note that the amount of solid $\mathrm{MoO}_{3}$ keep changing during the ramping process, which results in a gradient in the distribution of TMDC flakes grown on the substrate in a traditional HCVD process. 


\section{HCVD grown TMDC flakes show position dependent phenomenon on sapphire}

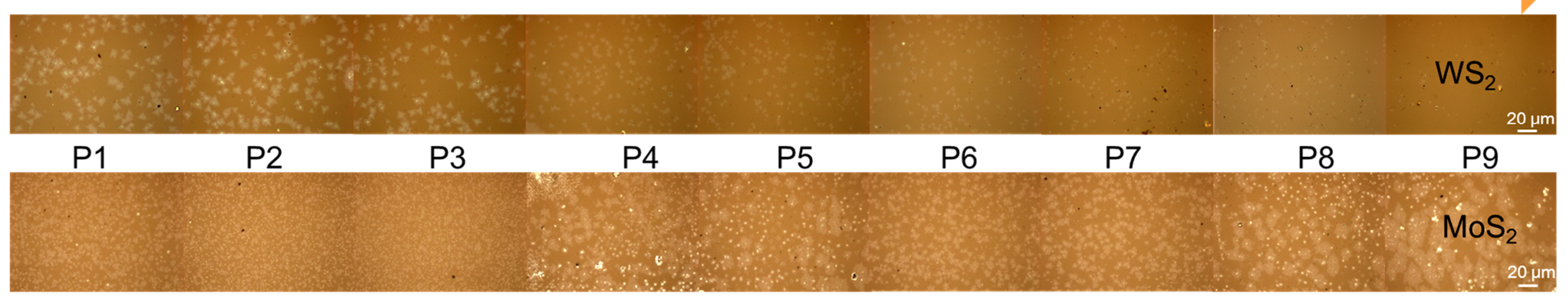

HCVD grown TMDC flakes show position dependent phenomenon on $\mathrm{SiO}_{2} / \mathrm{Si}$
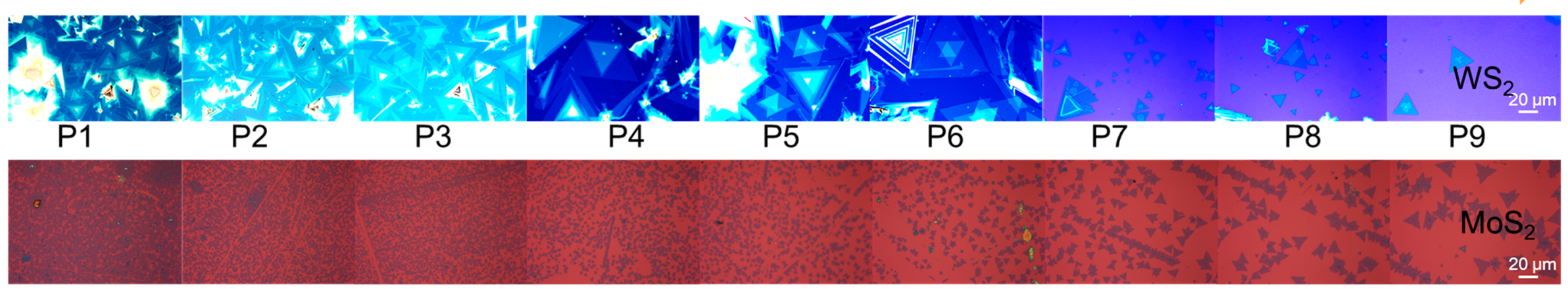

Figure S3. Typical optical images of as-grown TMDCs in a traditional HCVD system, which shows the position dependent distribution of flakes grown on both sapphire and $\mathrm{SiO}_{2} / \mathrm{Si}$ substrates due to the uncontrolled concentration, gas and temperature conditions. 


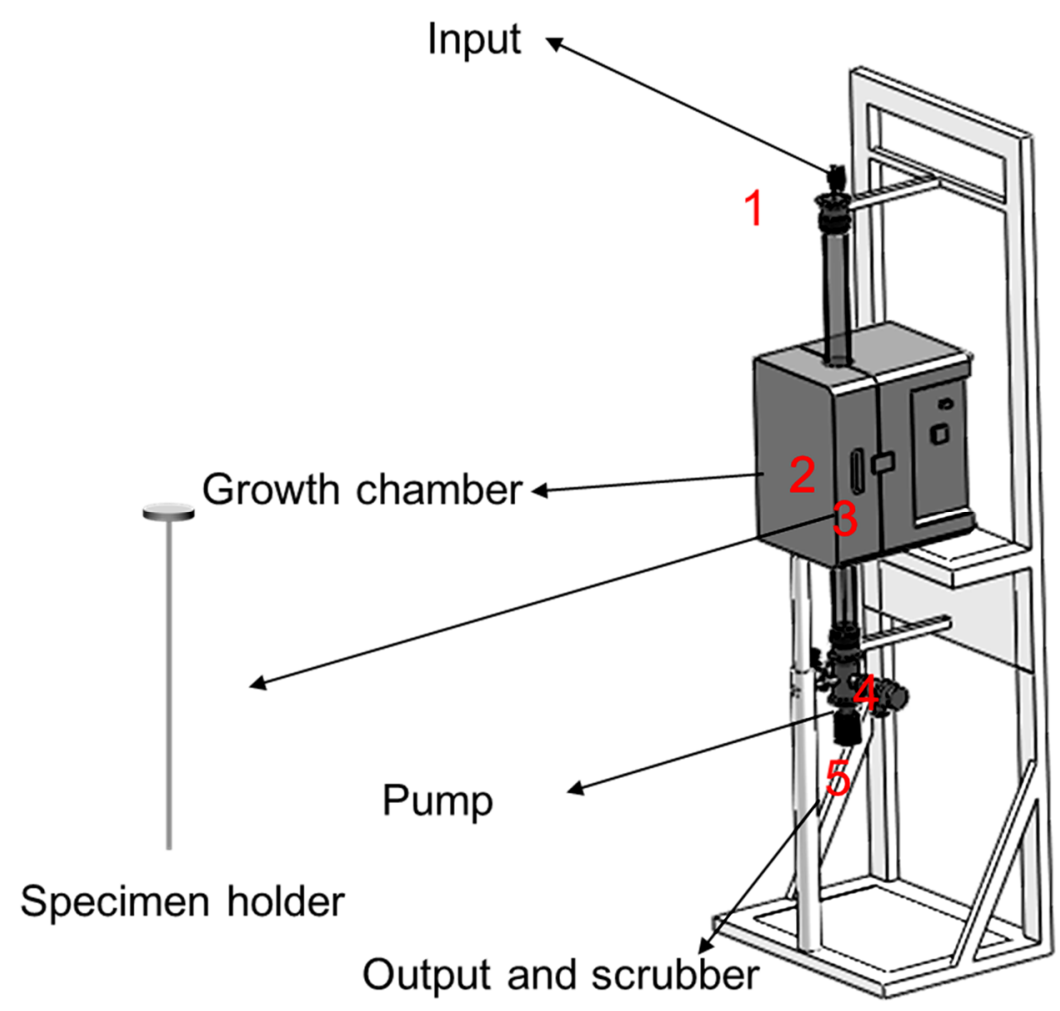

Figure S4. The homemade VCVD system that mainly composed of an input system, a growth chamber with specimen holder, an output system and a scrubber. Here, 1 is the input system for introducing the precursors, 2 is the growth chamber, 3 is the special specimen holder, 4 is the pump for removing the air, 5 is output and scrubber for filtering the post-reaction gases. 


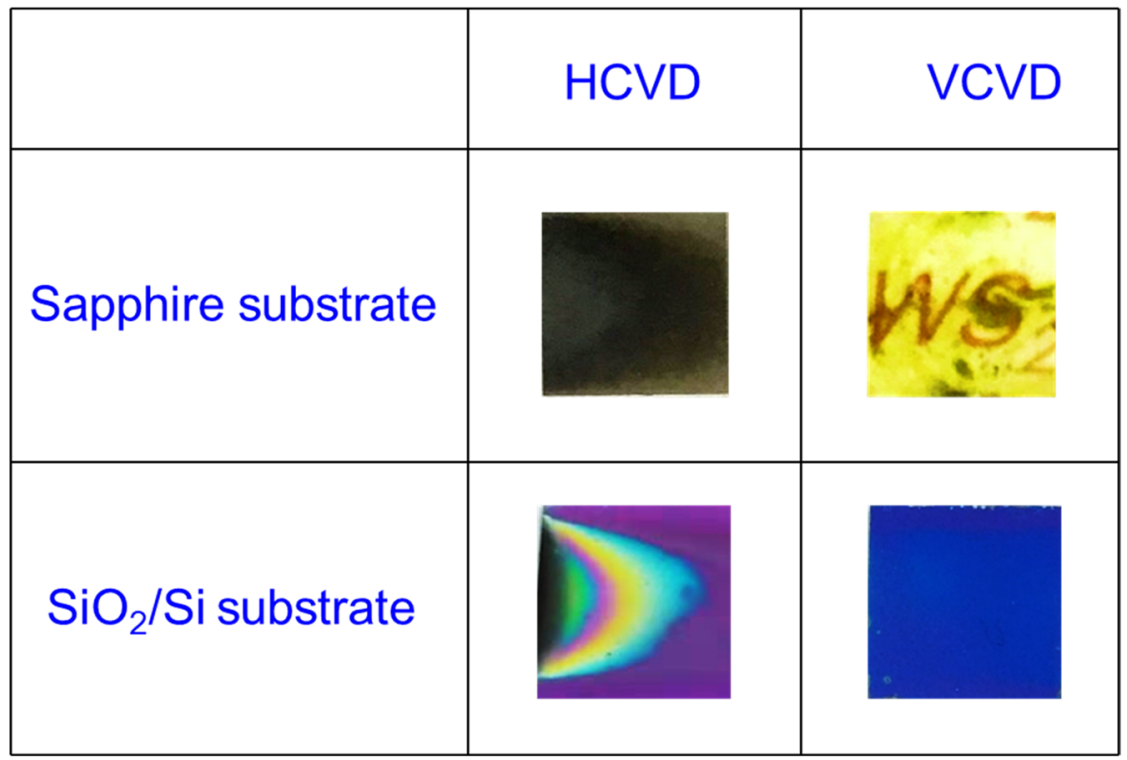

Figure S5. Comparison between HCVD and VCVD grown $\mathrm{WS}_{2}$ samples on sapphire and $\mathrm{SiO}_{2} / \mathrm{Si}$ substrates with an area of $10 \times 10 \mathrm{~mm}^{2}$. The former shows more a uniform color than the latter, which highlights the advantages of the VCVD system for the growth of highly-uniform TMDCs. 

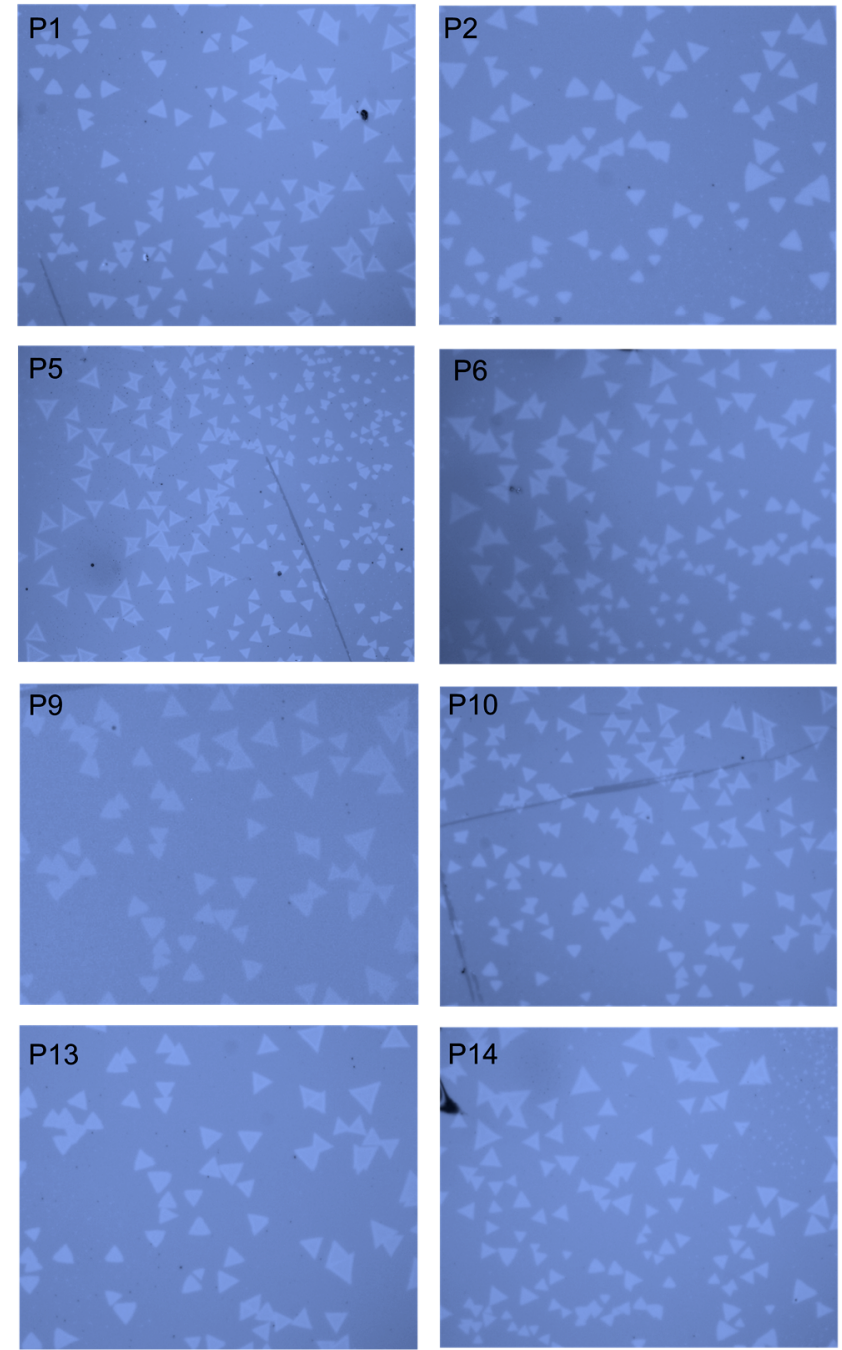
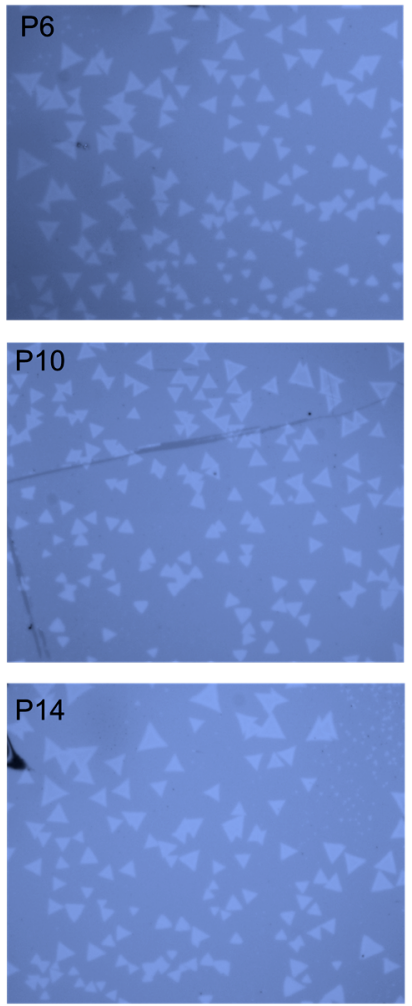

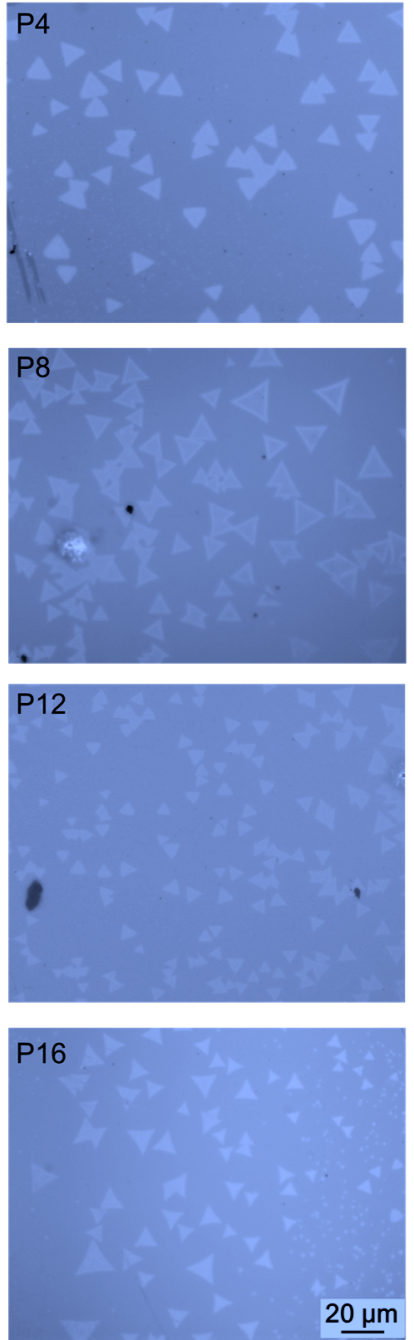

Figure S6. Optical images of VCVD-grown $\mathrm{WS}_{2}$ domains on a sapphire substrate taken from the 16 different positions, demonstrating the position-independent uniform distribution of $\mathrm{WS}_{2}$ domains across the whole substrate. 


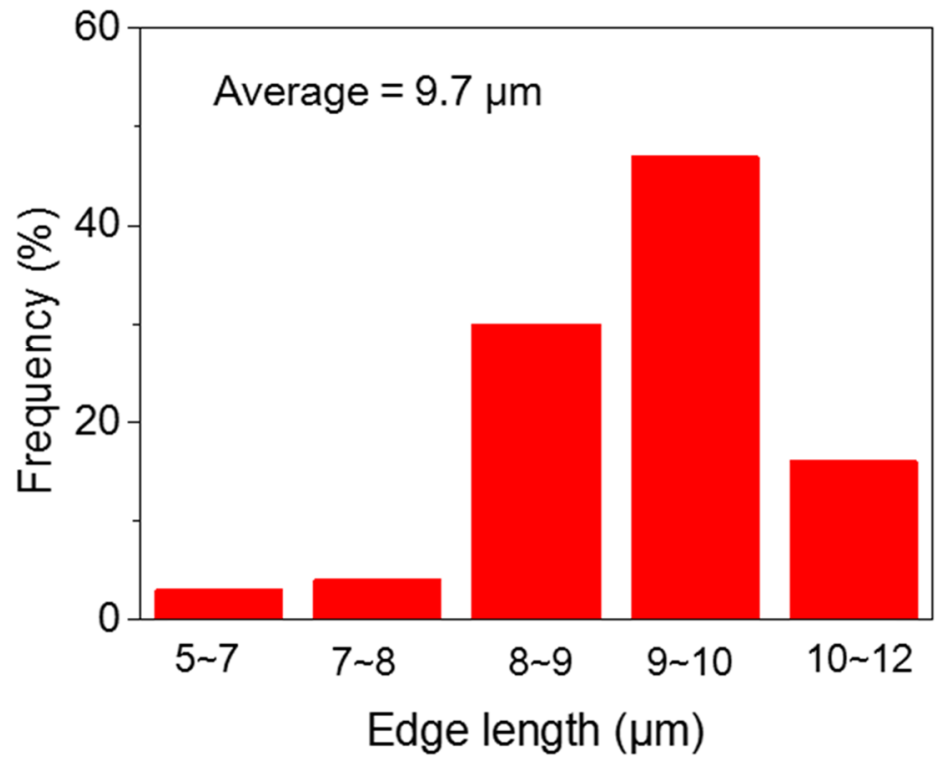

Figure S7. Statistics of the domain sizes of VCVD-grown $\mathrm{WS}_{2}$ show an average size of $9.7 \mu \mathrm{m}$.
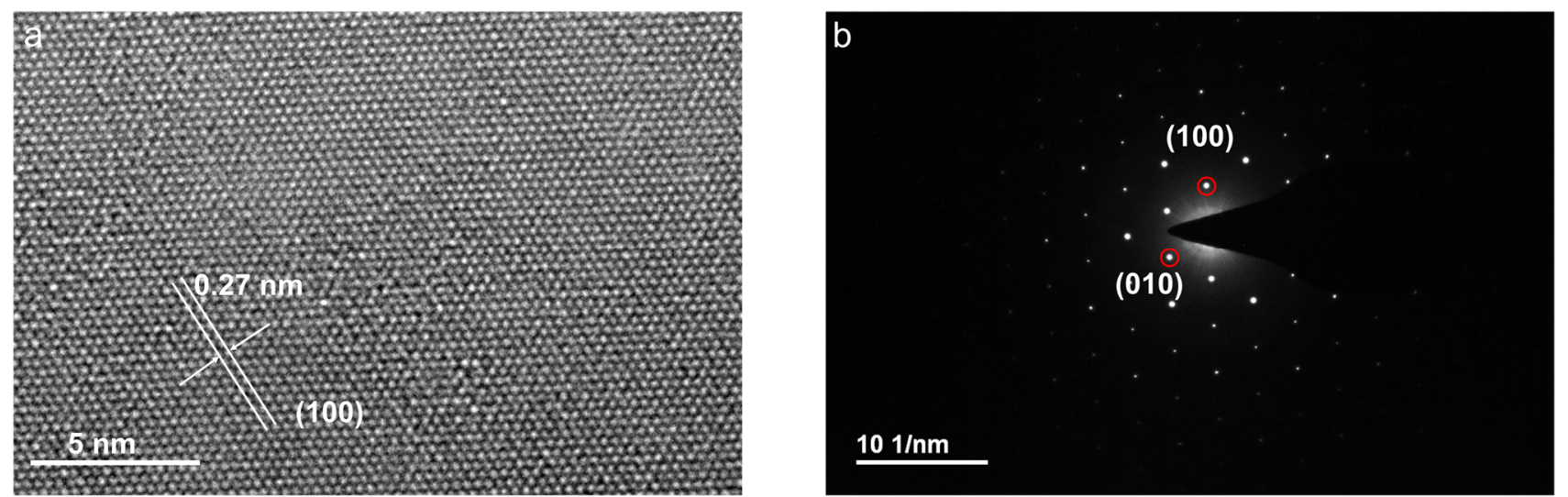

Figure S8. A typical HRTEM image of the VCVD-grown $\mathrm{WS}_{2}$ and its SAED pattern, showing the growth of high quality single crystalline flake. 
a

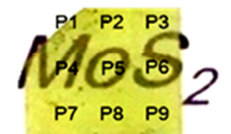

b
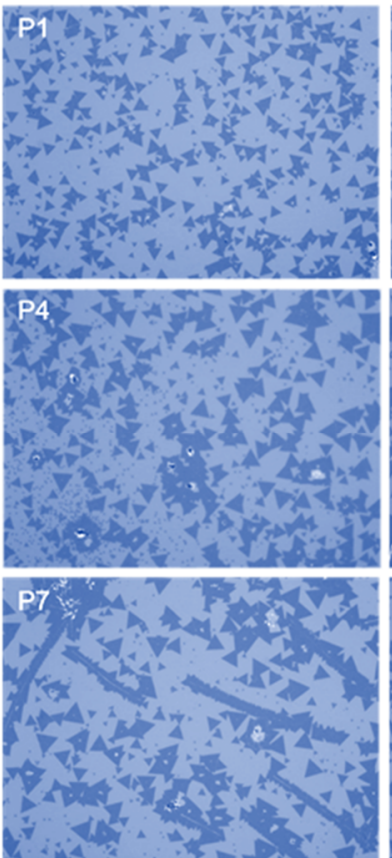
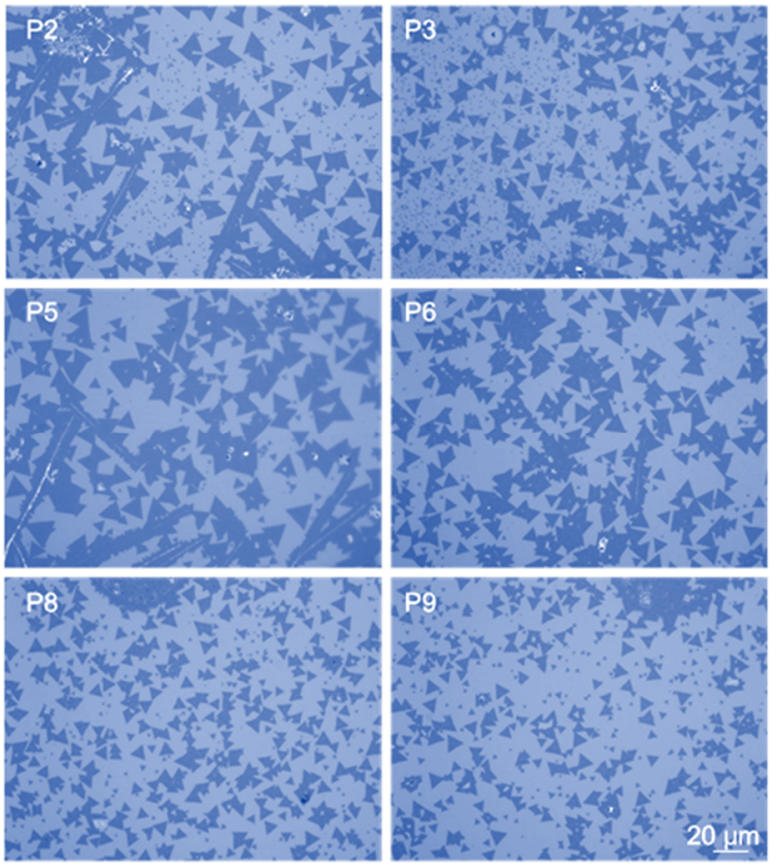

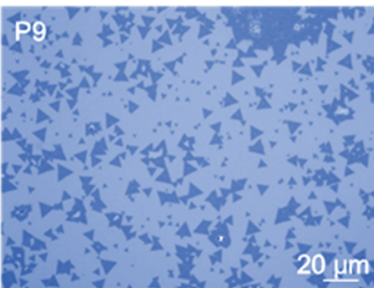

Figure S9. (a, b) Corresponding optical images of the as-grown $\mathrm{MoS}_{2}$ on a sapphire substrate show a position-independent distribution of TMDC domains, which verifies the universality of the VCVD design for the growth of highly-uniform 2D TMDCs. 
a
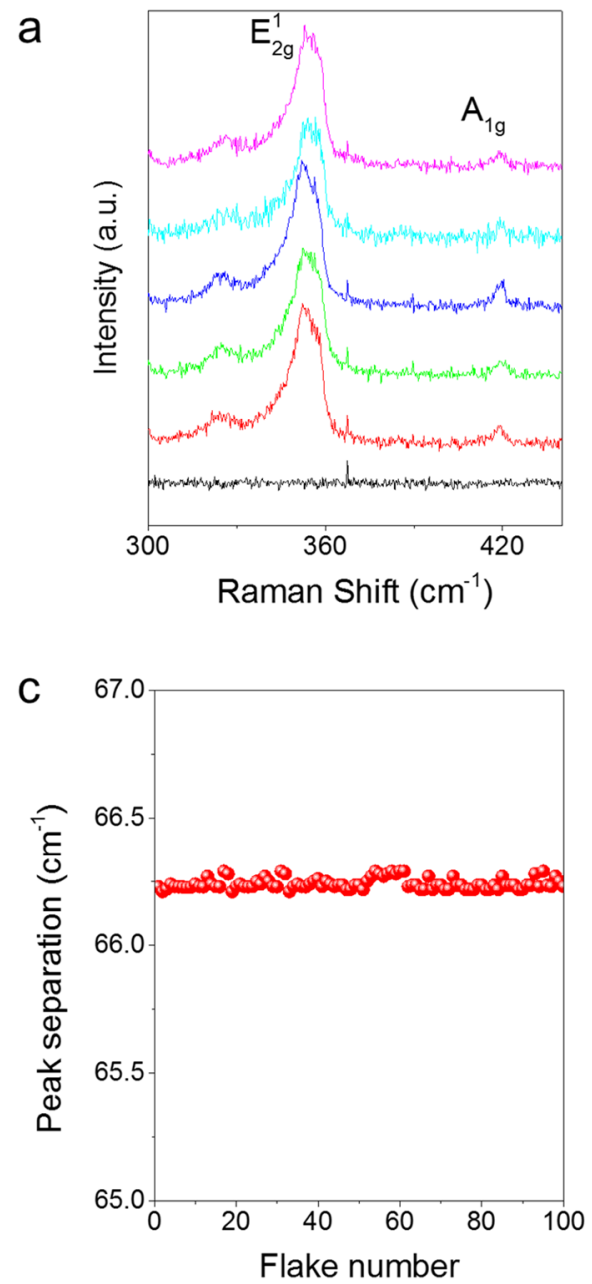

b
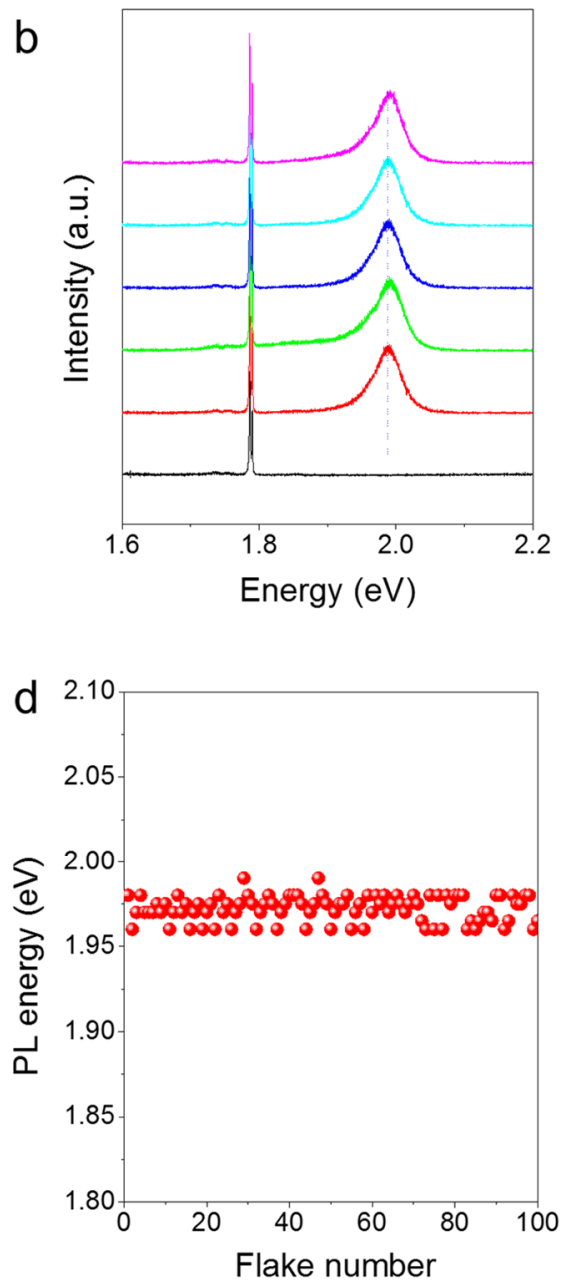

Figure S10. (a, b) Typical features of Raman and PL peaks for monolayer $\mathrm{WS}_{2}$ grown by the VCVD system on a sapphire substrate taken at five random positions. (c, d) The statistical values of peak separation of the $\mathrm{E}_{2 \mathrm{~g}}{ }^{1}\left(352 \mathrm{~cm}^{-1}\right), \mathrm{A}_{1 \mathrm{~g}}\left(419 \mathrm{~cm}^{-1}\right)$ and $\mathrm{PL}(1.98 \mathrm{eV})$ are plotted as a function of 100 different flakes. 

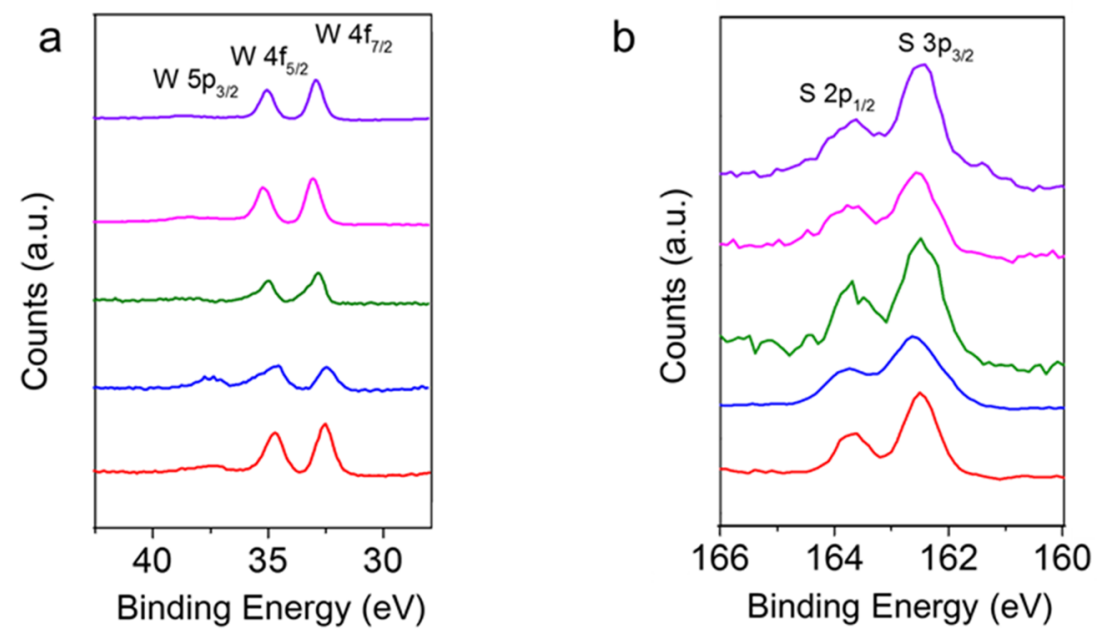

Figure S11. To identify the chemical composition of the VCVD-grown $\mathrm{WS}_{2}$, XPS was used to measure the binding energy of W and S. Figure S11a and S11b show the binding energy profiles for W 4f and $\mathrm{S} 2 \mathrm{p}$ at different positions. Three peaks at $33.67,35.86$, and $38.87 \mathrm{eV}$ are attributed to $\mathrm{W} 4 \mathrm{f}_{7 / 2}, \mathrm{~W} 4 \mathrm{f}_{5 / 2}$, and $\mathrm{W} 5 \mathrm{p}_{3 / 2}$, respectively. The peaks with binding energies of 162.48 and $163.60 \mathrm{eV}$ are assigned to $\mathrm{S}$ $2 p_{3 / 2}$ and $S 2 p_{1 / 2}$, respectively. All these results are consistent with the reported values for $\mathrm{WS}_{2}$ crystals.
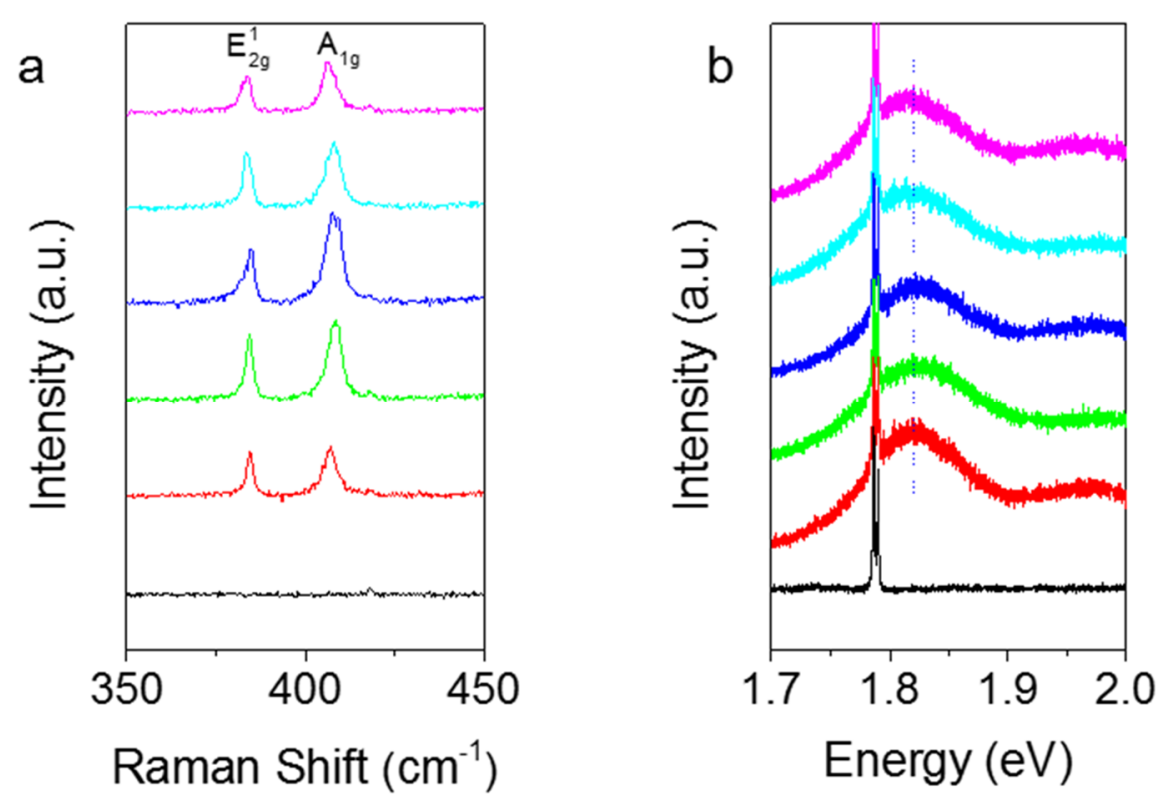

Figure S12. (a, b) Typical features of Raman and PL peaks for monolayer $\mathrm{MoS}_{2}$ grown on a sapphire substrate by VCVD with measurements taken at five random positions. 
a

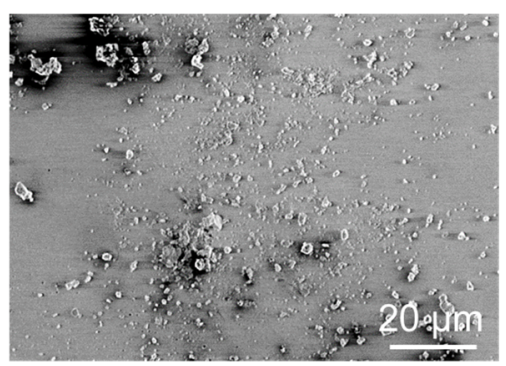

b

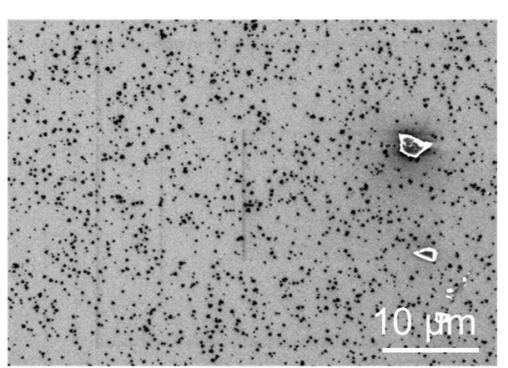

c

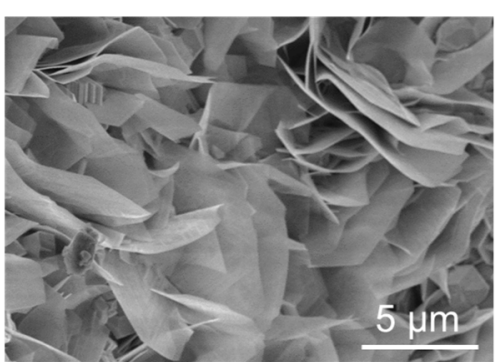

Figure S13. TMDCs grown in a HCVD system with substrate perpendicular to the horizontal gas flow direction. The SEM images show the formation of different morphologies at different positions, such as clusters, particles and vertically-standing TMDC nanoflowers, which indicates the poor controllability and bad uniformity of this design.

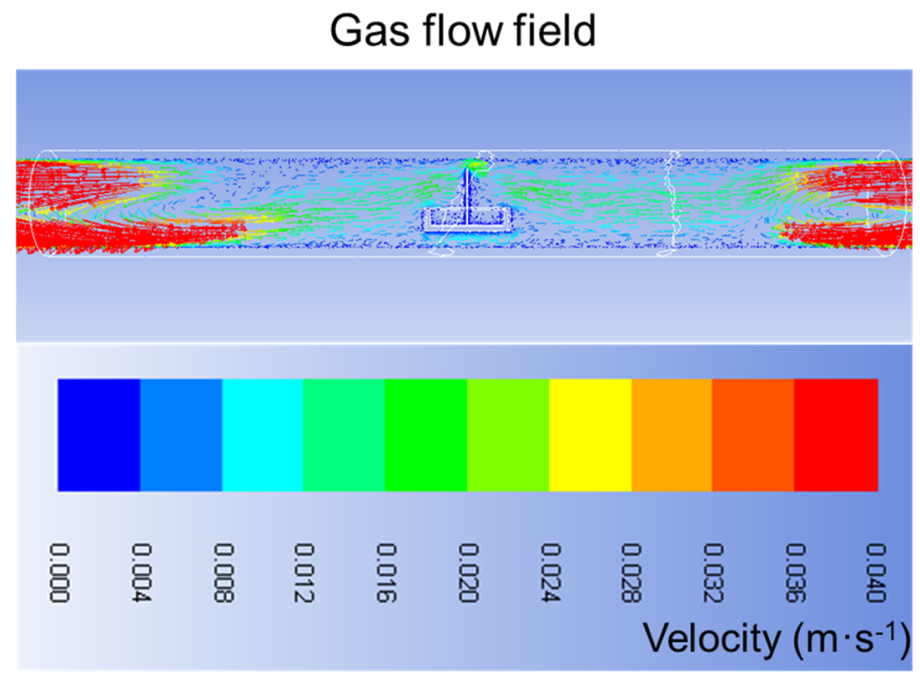

Figure S14. CFD simulation of the distribution of gas flow velocity in HCVD systems with a perpendicular substrate, which demonstrates the formation of vortexing gas of precursor concentration along the substrate during the CVD growth process. 


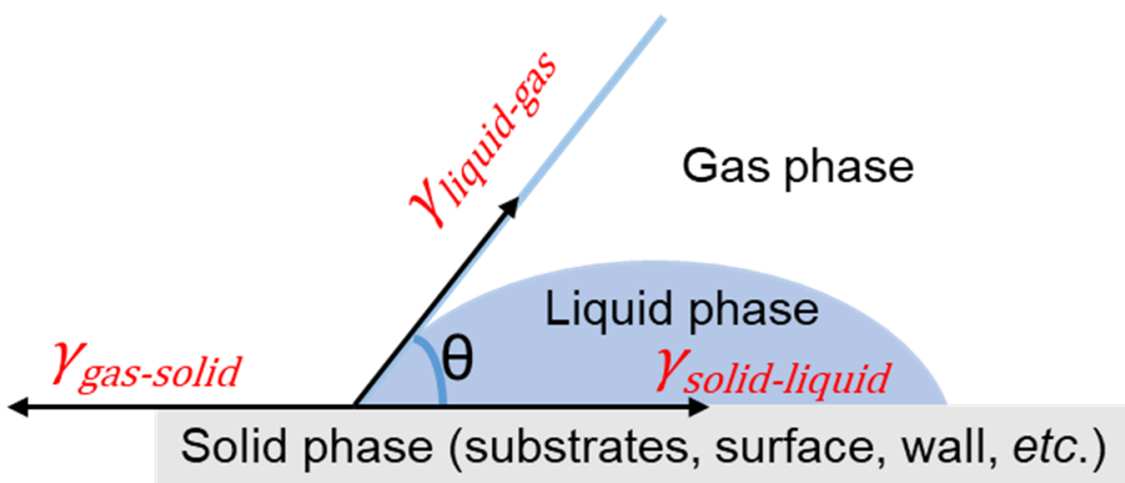

Figure S15. Schematic representation of a cap-shaped nucleus and showing an included angle $(\theta)$ which is known as the contact angle. According to Young's Equation:

$$
\gamma_{\text {gas-solid }}=\gamma_{\text {solid-liquid }}+\gamma_{\text {liquid-gas }} \cos \theta \quad(0 \leqslant \cos \theta \leqslant 1)
$$

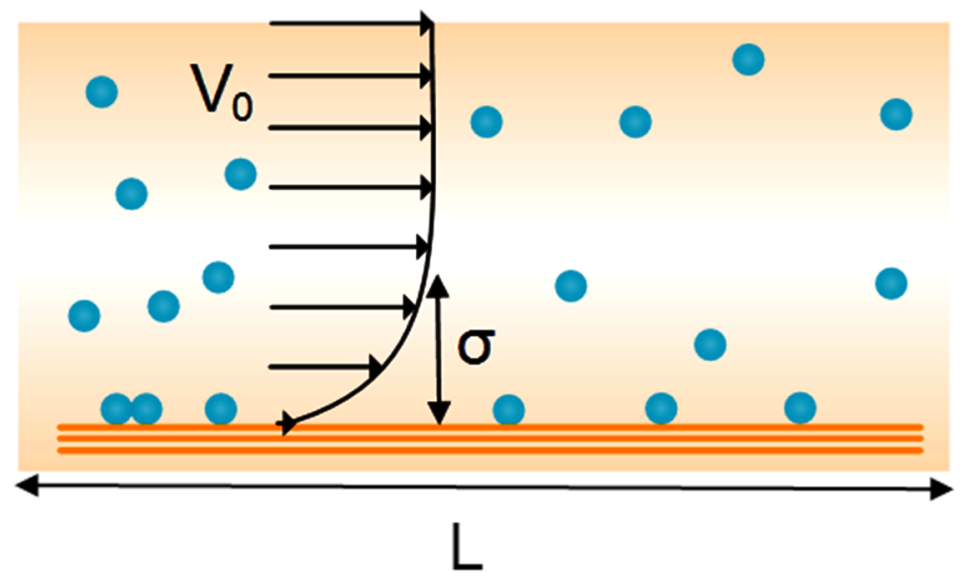

Figure S16. Schematic showing viscous laminar flow in the HCVD system and related physical parameters. It shows the formation of a stagnant layer above the substrate surface. Here, $\sigma$ is the average thickness of the stagnant layer, $\mathrm{L}$ is the length of the substrate, and $\mathrm{V}_{0}$ is the flow velocity. Differences in $\mathrm{V}_{0}$ mean that the gas flow of the precursor is not uniform in the furnace, and the flow rate at the center is higher than at the edge. 


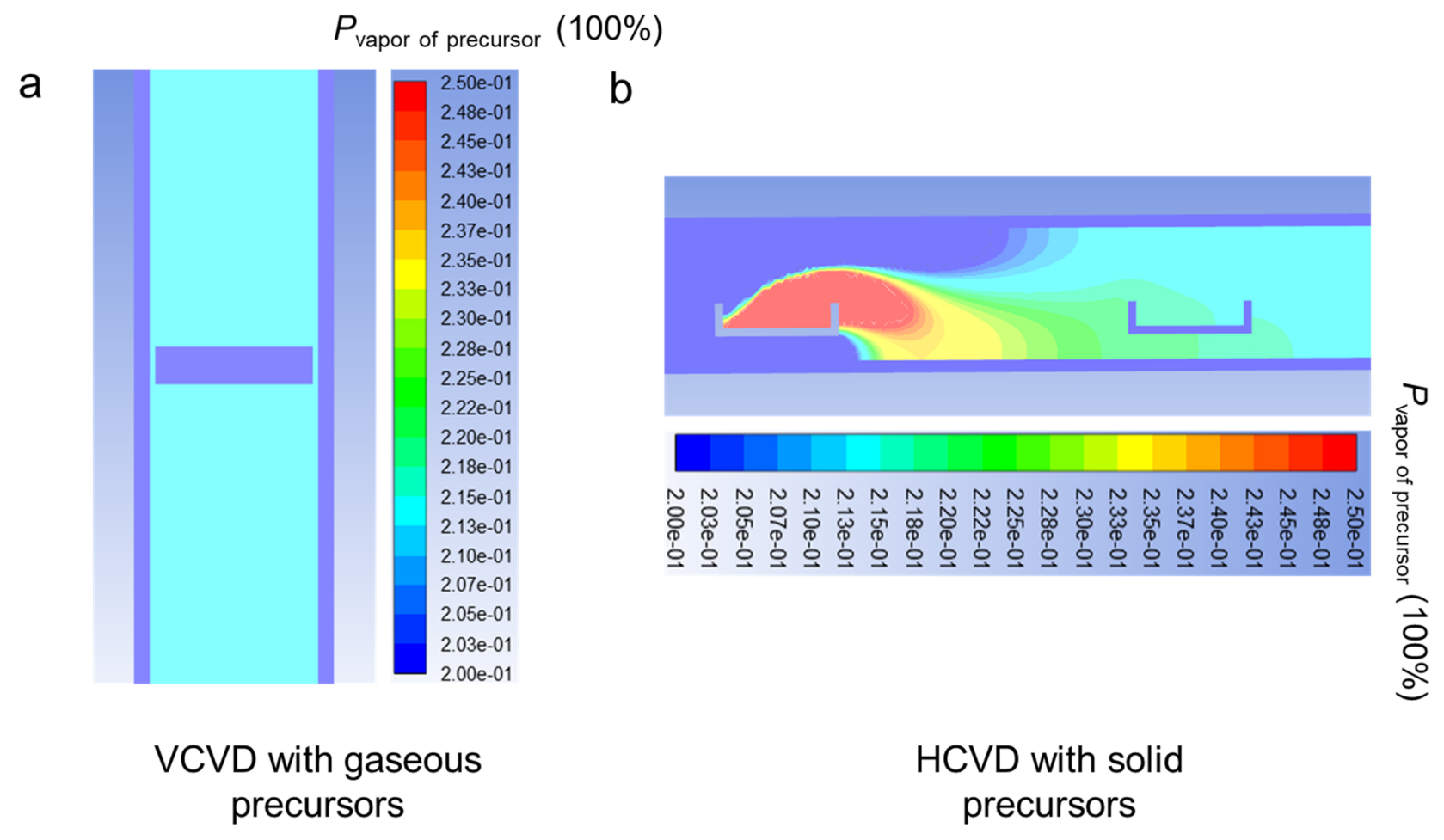

Figure S17. CFD simulations of the distribution of partial pressure of vapor precursors in VCVD (a) and HCVD (b) systems. 